\title{
Imaging magnetic responses of nanomagnets by XPEEM
}

\author{
O. Sandig a,b J. Herrero-Albillos a,d,e , F.M. Römer ${ }^{c}$, N. Friedenberger ${ }^{c}$, \\ J. Kurde ${ }^{\mathrm{b}}$, T. Noll ${ }^{\mathrm{a}}$, M. Farle ${ }^{\mathrm{c}}$, F. Kronast ${ }^{\mathrm{a}, *}$ \\ a Helmholtz Zentrum Berlin für Materialien und Energie, Albert-Einstein-Str. 15, 12489 Berlin, Germany \\ ${ }^{\mathrm{b}}$ Freie Universität Berlin, Arnimallee 14, 14195 Berlin, Germany \\ c Universität Duisburg-Essen, Lotharstr.1, 47048 Duisburg, Germany \\ ${ }^{\mathrm{d}}$ Centro Universitario de la Defensa, Ctra. de Huesca s/n, E-50090 Zaragoza, Spain \\ e Instituto de Ciencia de Materiales de Aragón and Departamento de Física de la Materia Condensada, CSIC - Universidad de Zaragoza, Pedro Cerbuna 12, E-50009 Zaragoza, Spain
}

\section{A R T I C L E I N F O}

\section{Article history:}

Available online $\mathrm{xxx}$

\section{Keywords:}

XPEEM

Magnetic nanostructures

Microscopy

Magnetic susceptibility

\begin{abstract}
A B S T R A C T
The Spin-resolved Photoelectron Emission Microscope (SPEEM) is a permanently installed set-up at Helmholtz-Zentrum Berlin (HZB). Due to its specific contrast it is mainly used for magnetic imaging and micro-spectroscopy with quantitative analysis. A crucial point in magnetic imaging is the application of magnetic fields. Many experiments require observation of magnetic responses or the preparation of a certain magnetic state during the measurement. We present a dedicated magnetic sample holder combining magnetic field during imaging with additional temperature control. This set-up enables SPEEM to measure magnetization curves of individual Fe nanocubes $(18 \mathrm{~nm})^{3}$ in size. If additionally alternating magnetic fields are applied we can image the local magnetic AC susceptibility $\left(\chi_{\mathrm{AC}}\right)$ as a function of temperature. The latter is ideally suited to visualize local variations of the Curie temperature $\left(T_{\mathrm{C}}\right)$ in nanoand microstructures.
\end{abstract}

(C) 2012 Elsevier B.V. All rights reserved.

\section{Introduction}

A fundamental question in magnetic systems with reduced dimensions is how the magnetic properties depend on the length scale of lateral confinement. In the case of magnetic nanoparticles in particular, magnetic properties might not only depend on their size and shape, they additionally can depend on their chemical composition, their orientation and configuration. Disentangling such complex interplay of those different factors often requires spatially resolved investigations with element and magnetization sensitive techniques. Over the recent years photoelectron microscopy in combination with synchrotron light (XPEEM) became a well-established imaging technique [1], and nowadays XPEEMs are available at most synchrotron facilities. Its spatial resolution, of $30-100 \mathrm{~nm}$, in combination with element specific contrast is ideally suited for element resolved imaging and quantitative micro-spectroscopy of magnetic nanoparticles. If the polarization of the X-rays is variable, the magnetic circular dichroism (XMCD), and the magnetic linear dichroism (XMLD) can be employed for magnetic imaging [2-4]. By application of the sum rules even spin and orbital momentum can be quantified [5]. Combining XPEEM with other imaging techniques of higher spatial resolution, e.g. atomic force microscopy (AFM) or scanning

\footnotetext{
* Corresponding author.

E-mail address: Florian.kronast@helmholtz-berlin.de (F. Kronast).
}

electron microscopy (SEM) we can investigate magnetic properties of nanoparticles much smaller than the resolution limit of XPEEM $[6,7]$. In addition to that, recently emerging XPEEM instruments with aberration correctors promise to boost the spatial resolution of XPEEM into a regime well below $10 \mathrm{~nm}[8,9]$. Even dimensionality could recently be added to XPEEM. Placing the sample on a dielectric multilayer mirror, depth resolved XPEEM can be performed via standing wave excitation $[10,11]$. For free standing three dimensional nanostructures bulk and surface magnetization can be imaged simultaneously by evaluating the transmitted signal from the object's shadow [12]. Besides those obvious advantages, the difficulty of applying magnetic fields during imaging is certainly a major drawback of the XPEEM technique compared to other $\mathrm{X}$-ray based techniques such as X-ray microscopy [13] or scanning $X$-ray microscopy [14]. But in turn these techniques require samples transparent to X-rays which is not always possible. To overcome the limitations of XPEEM magnetic sample holders have been designed, allowing for preparation of certain magnetic states during measurement [15] or imaging the magnetic response of a sample on a strip line during and after a current pulse [16,17]. At the Spin-resolved Photoelectron Emission Microscope (SPEEM) at Helmholtz-Zentrum Berlin (HZB) we use a custom made magnetic sample holder to image the magnetic response of the sample in an applied magnetic field. Applying laterally confined magnetic fields due to a special yoke geometry minimizes the deflection of photoelectrons. In many cases the extension of the magnetic field is only a few times larger than the microscope's field of view. In this set-up 
we can apply magnetic fields of several tens of mT during imaging, without affecting the spatial resolution. An additional built-in temperature control allows performing magnetic field and temperature dependent XPEEM studies of magnetic nanostructures. In combination with the above mentioned possibilities this strongly extends the capabilities of the SPEEM set-up making it a dedicated instrument for investigation of magnetic micro and nanostructures. In the following we will give a description of the set-up and present two representative applications for magnetic imaging in combination with magnetic field and temperature control.

\section{Experimental set-up}

The SPEEM set-up is based on an Elmitec PEEM III with energy analyzer. It is permanently installed at the BESSY II storage ring at HZB. A unique feature of that instrument is its possibility to perform spin-resolved micro-spectroscopy by mini-Mott detectors attached to the instrument $[18,19]$. The X-ray source is an elliptical undulator with full polarization control (UE49). The photon energy can be tuned between 80 and $1800 \mathrm{eV}$. The monochromator is a plane grating monochromator (PGM) with an energy resolution $(E / \Delta E)$ of $10^{4}$ at $700 \mathrm{eV}$. An ellipsoidal mirror focuses the X-ray beam with a spot size of $8 \mu \mathrm{m} \times 20 \mu \mathrm{m}$ onto the sample. The manipulator of the SPEEM is equipped with liquid Nitrogen cooling and azimuth rotation. Further details about the SPEEM set-up are published elsewhere [19].

The layout of the magnetic sample holder with temperature control is shown in Fig. 1a. Its base plate (magenta color) follows the Elmitec design with four electrical contacts at its backside. The monolithic magnetic yoke (blue color) is thermally isolated from the base plate by four ceramic plates. Two symmetric coils are used to generate the magnetic field (orange color). The sample is placed on top of the yoke clamped by an aluminum capping plate (green color). Similar to the write head of a magnetic tape recorder, the yoke sits behind the sample and the magnetic field lines close through the sample surface. Depending on the gap width and the sample thickness we can apply magnetic fields up to $50 \mathrm{mT}$ during imaging. Due to the laterally confined magnetic field of our sample holder, the deflection of photoelectrons is minimized and can be corrected by the deflectors of the objective lens. Meanwhile, lens tracking has been implanted in the SPEEM measurement software, that automatically corrects image shifts during a field sweep. With such automatic lens tracking magnetic switching processes can even be observed online.

Furthermore, temperature control is integrated in the sample holder. The magnetic yoke has a central bore in which a resistive heater can be placed (see the arrow in Fig. 1b). A Pt100 resistive thermometer is attached to the backside of the yoke for temperature monitoring. To minimize the thermal drift of the manipulator during temperature changes the manipulator is cooled with a constant flow of liquid Nitrogen, while the sample temperature is set with the internal heating of the yoke. Due to the thermal isolation between yoke and base-plate the thermal drift of the manipulator is very small during temperature changes. The temperature range is about $115-430 \mathrm{~K}$. Several types of magnetic sample holders with varying gap sizes ( $50 \mu \mathrm{m}-1.5 \mathrm{~mm}$ ) have been built. In the following example we demonstrate imaging in applied magnetic fields up to $23 \mathrm{mT}$ using a sample holder with $500 \mu \mathrm{m}$ gap size. Higher magnetic fields can be applied reducing the gap of the magnetic yoke. Alternating magnetic fields with frequencies up to $250 \mathrm{~Hz}$ can be applied as well. It has been demonstrated that with these sample holders, combining magnetic field and temperature control, spatially resolved magnetic AC susceptibility $\left(\chi_{\mathrm{AC}}\right)$ can be investigated by XPEEM [20]. The magnetic field can also be combined with additional electrical contacts at the sample surface, e.g. for field dependent transport measurements.

\section{Examples}

\subsection{Nanoparticles}

In our first example we demonstrate the possibility to image magnetic responses of individual nanoparticles by XPEEM. Within structures on the nanometer scale magnetic properties are influenced by finite size effects which can change the effective magnetization. For example nanoparticles behave(super)paramagnetic rather than ferromagnetic at room temperature, or collections of particles behave superferromagnetic depending on their arrangement and magnetic interaction [21]. Recording the magnetic hysteresis of individual nanoparticles we can directly determine their coercivity and magnetization at remanence. Furthermore, from the shape of the hysteresis we can estimate the magnetic anisotropy energy. These important parameters for possible applications in data storage or sensor devices are very difficult to measure in randomly oriented particle ensembles due to their orientation and configuration dependence. E.g. dipolar coupling between neighboring particles can strongly influence their magnetic properties [7]. An example of a magnetic hysteresis of an individual nanoparticle recorded by XPEEM at low temperature (115 K) is shown in Fig. 2. Panel (a) shows a XPEEM image of an array with mostly single Fe nanocubes $(18 \mathrm{~nm})$ in size. Size and position of individual nanocubes is identified by matching the XPEEM image with the high resolution scanning electron microscopy (SEM) micrograph of the same sample area displayed in panel (b). Due to the low particle density interparticle interactions can be neglected at this site. The XPEEM image is part of an image stack, taken at different magnetic fields. Each image in the stack represents the average of about 600 individual XPEEM images that have been drift corrected and co-added. Each image was recorded with an exposure time of $1 \mathrm{~s}$. The magnetic hysteresis corresponding to the indicated nanocube is displayed in the upper panel of Fig. 2c. To record magnetization curves we exploited the magnetic XMCD contrast at the $\mathrm{Fe}_{3}$ resonance. At each magnetic field we recorded XPEEM images with opposite helicity. To extract the hysteresis of an individual Fe nanocube we integrated the intensity from a particular region of interest containing the whole particle and evaluated the local XMCD signal, i.e. the difference of the local intensity of the two helicities divided by their sum. A neighboring region of interest containing no particle was used to perform a local background subtraction. In comparison to [7] the observed hysteresis at $115 \mathrm{~K}$ is rectangular shaped with almost full magnetization at remanence for a single Fe nanocube. Obviously $115 \mathrm{~K}$ lies below the blocking temperature $\left(T_{\mathrm{B}}\right)$ of that single Fe nanocube.

To evidence the influence of the applied magnetic field on the spatial resolution of the SPEEM, we evaluated the width of two orthogonal line profiles across a single Fe nanocube for every magnetic field (red and blue lines in Fig. 2a). The intensity along the line profiles is shown as an inset. The lower panel of Fig. 2c displays the full width at half max (FWHM) of a Gauss fit to the line profiles at different fields. Assuming that the measured average FWHM of about $46 \mathrm{~nm}$ is a convolution of the instrumental resolution and the particle size $(18 \mathrm{~nm})$ we obtain a spatial resolution of about $42 \mathrm{~nm}$. Considering that each image represents the average of about 600 XPEEM images, recorded during liquid Nitrogen cooling, which introduces additional vibrations, $42 \mathrm{~nm}$ is a very reasonable spatial resolution for XPEEM. Apart from some scatter due to the varying alignment skills of the operator (during that measurement the automatic lens tracking has not been installed yet), Fig. 2c does not show any correlation between the FWHM of the 
(a)

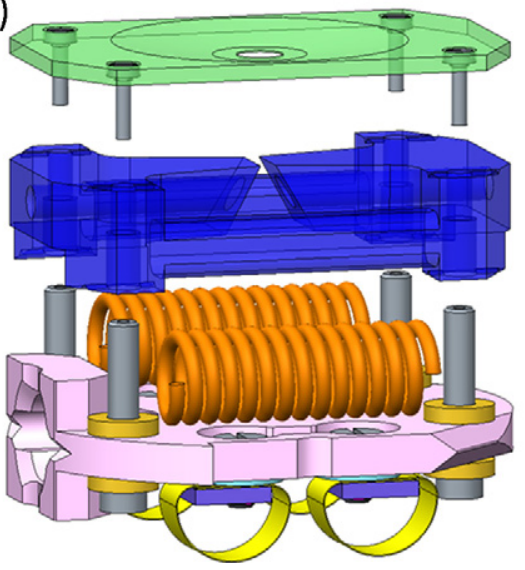

(b)

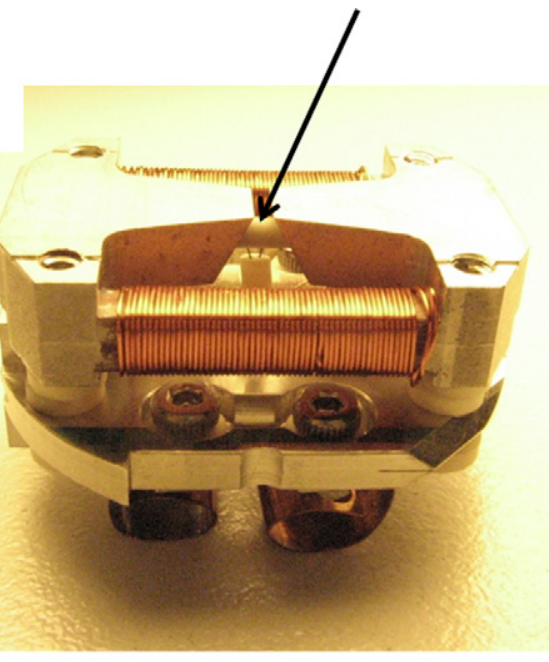

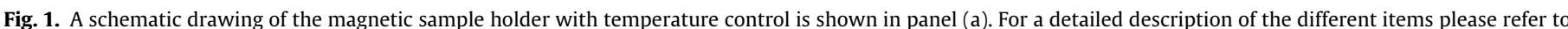

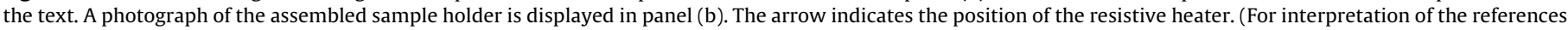
to color in the text, the reader is referred to the web version of the article.)

line profile and the applied magnetic field. Obviously the spatial resolution is not affected by an applied magnetic field up to $23 \mathrm{mT}$. The only noticeable field dependent effect is a slight diagonal image distortion.

\subsection{Local AC susceptibility}

The second example demonstrates how imaging the local AC susceptibility $\chi_{\mathrm{AC}}$ by XPEEM can visualize the influence of nano- or micro-structuring on the local magnetic stability. Finite size effects can change the effective magnetization and reduce the Curie temperature $\left(T_{\mathrm{C}}\right)$ dramatically (see for example Refs. [7,22]). These changes of $T_{C}$ are of increasing interest for the stability of data storage and spintronic applications. A question in ferromagnetic systems with reduced dimensions is, whether the magnetic properties laterally vary at the edges of the sample, at defects or at steps due to the underlying substrate. In the present example we use element-specific AC susceptibility measurements by XPEEM to investigate local variations of $T_{C}$ in a patterned magnetic film and compare it with a continuous film of identical thickness. To grow well defined magnetic structures of different shape and size, we used pre-patterned Si substrates on which we
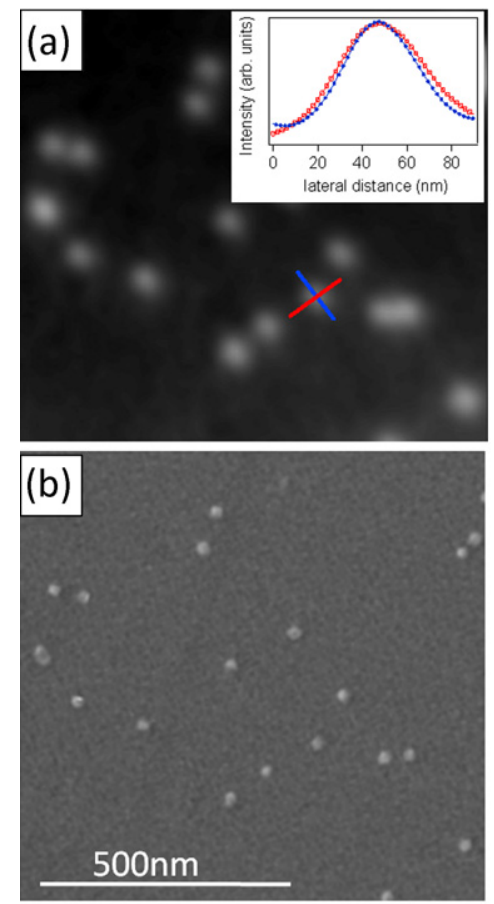

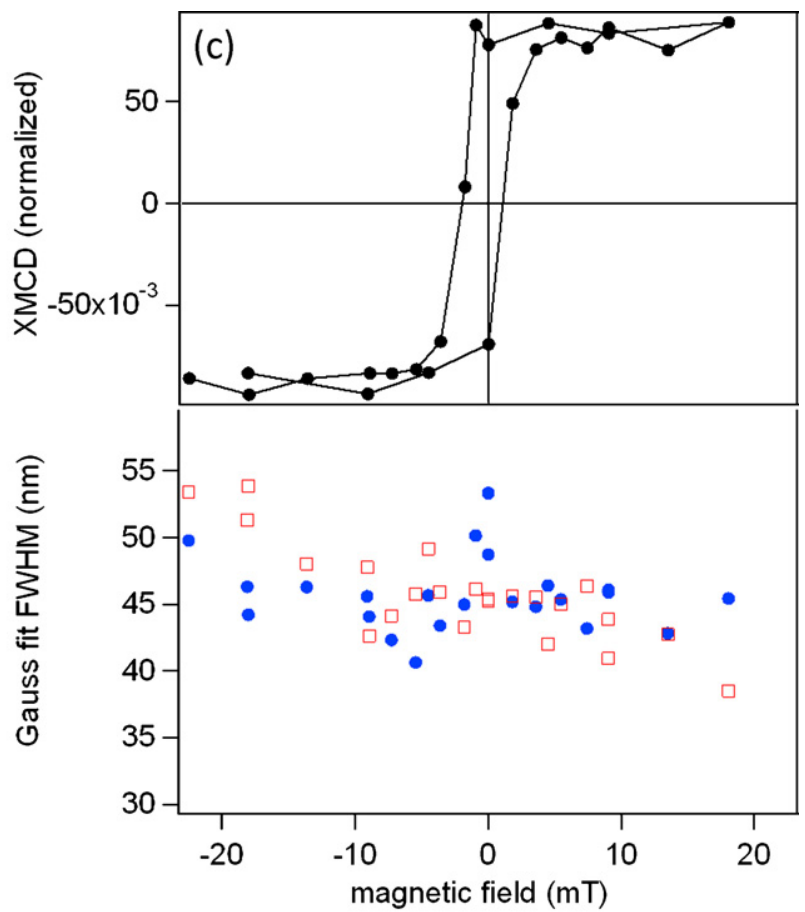

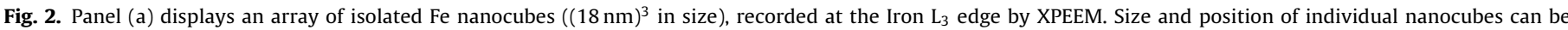

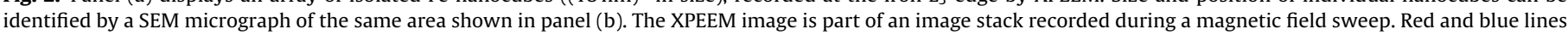

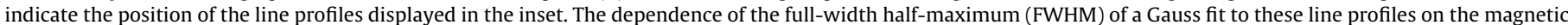

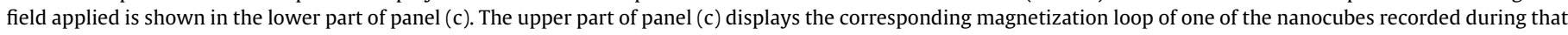
magnetic field sweep. (For interpretation of the references to color in this figure legend, the reader is referred to the web version of the article.) 

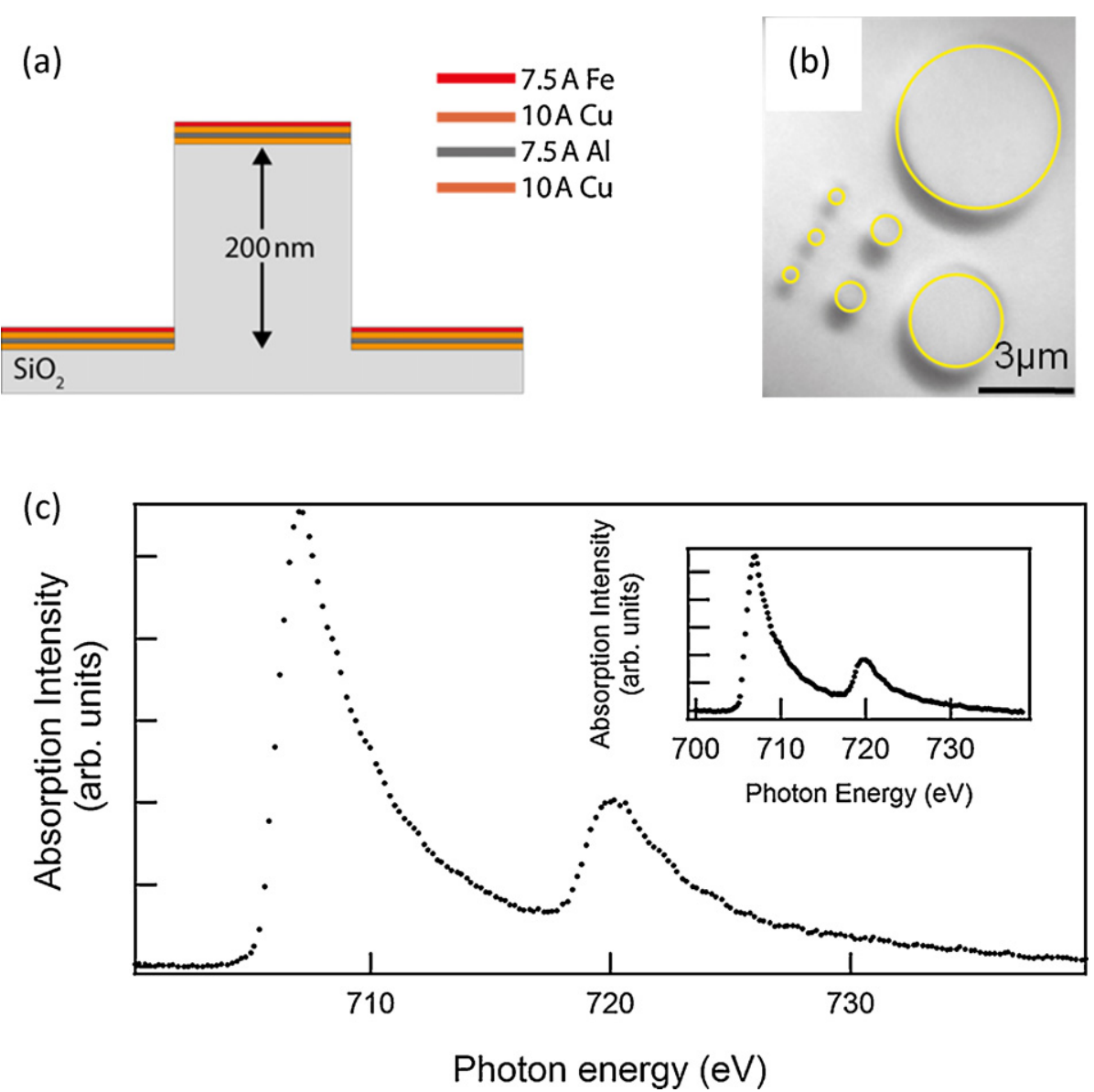

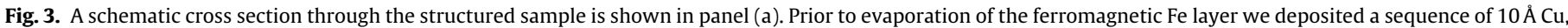

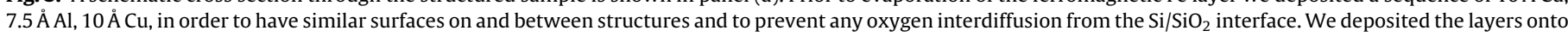

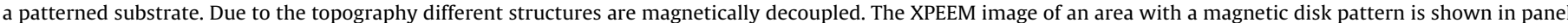

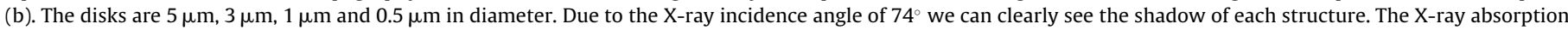
spectra recorded at the Iron $\mathrm{L}_{2,3}$ edges directly after deposition and 3 days later (inset) exhibit no sign of oxidation.

deposited ultrathin Fe films in the SPEEM preparation chamber. The structured substrates were prepared using electron beam lithography in combination with reactive ion etching. A schematic layout of our sample is shown in Fig. 3a. The height difference between the structures and the surrounding substrate is about $200 \mathrm{~nm}$. The different structures and the surrounding film are magnetically decoupled from each other due to the sample topography. We tuned the thickness of the ferromagnetic layer to obtain ferromagnetic structures with a $T_{C}$ in the range between $110 \mathrm{~K}$ and $450 \mathrm{~K}$, which is accessible in the SPEEM set-up. To avoid any contamination of the magnetic layer and to obtain identical surface roughness in all areas, we grew additional buffer and protection layers. X-ray absorption spectra, taken at the Iron $\mathrm{L}_{3}$ and $\mathrm{L}_{2}$ edges, directly after deposition (Fig. 3c) and after the measurement (72 $\mathrm{h}$ later) (inset in Fig. 3c) show no sign of oxidation.

We investigated local variations of $T_{C}$ resulting from several structures of different sizes by imaging the local magnetic $\mathrm{AC}$ susceptibility $\left(\chi_{\mathrm{AC}}\right)$ as a function of temperature. The temperature is monitored by the Pt100 thermometer on our sample holder. Absolute values may differ by $10 \mathrm{~K}$, but the relative accuracy is about $0.2 \mathrm{~K}$. To measure $\chi_{\mathrm{AC}}$ we applied an alternating magnetic field (AC field) with $0.15 \mathrm{mT}$ amplitude at a frequency of $81 \mathrm{~Hz}$ (frequencies up to $250 \mathrm{~Hz}$ should be possible). The imaging unit of the SPEEM microscope was synchronized to the alternating field by gating the channel plate voltage with a voltage offset of $400 \mathrm{~V}$. For magnetic imaging we exploited the X-ray magnetic circular dichroism (XMCD) at the $\mathrm{Fe}_{2}$ resonance. In the images presented in the following, the $\chi_{\mathrm{AC}}$ signal is defined as the difference of two images taken at a fixed X-ray helicity but with opposite directions of the applied magnetic field divided by their sum.

Fig. 4 displays the local magnetic susceptibility $\chi_{\mathrm{AC}}$ of a patterned sample area at selected temperatures covering the range of the magnetic phase transition. The corresponding XPEEM image of that area is shown in Fig. 3b. It is displaying the local pattern, disks of $5 \mu \mathrm{m}, 3 \mu \mathrm{m}, 1 \mu \mathrm{m}$ and $0.5 \mu \mathrm{m}$ in diameter. Due to the height profile the shadow of each structure appears in this image as a dark area. In those shadow areas we find no AC signal and therefore they appear bright in Fig. 4. We also find an obvious contrast between the AC signal of the structures and that of the surrounding film, the contrast inverts between temperatures of $182 \mathrm{~K}$ and $213 \mathrm{~K}$. This already indicates different $T_{C}$ for the patterned area and the continuous film. To quantify the local variation of $T_{\mathrm{C}}$ between the continuous film and the $3 \mu \mathrm{m}$ disk we extracted the local magnetic susceptibility $\chi_{\mathrm{AC}}$ by integrating over a defined region of interest at each temperature. The local magnetic susceptibility $\chi_{\mathrm{AC}}$ and the corresponding areas are displayed in Fig. $5 \mathrm{a}$. We determined $T_{\mathrm{C}}$ by fitting the inverse of the magnetic susceptibility $\chi_{\mathrm{AC}}$ in the paramagnetic phase with the Curie-Weiss law $(1 /(\chi(T)))=(1 / C)\left(T-T_{\mathrm{C}}\right)$, where $C$ is the Curie constant $C=\left(1 / 3 k_{\mathrm{B}}\right) \mu_{0} n \mu^{2}, \mu$ is the magnetic moment per atom and $n$ the particle density per volume. This procedure avoids any influence in the determination of $T_{C}$ coming from magnetic domains, which might be present in the ferromagnetic phase. The inverse magnetic susceptibility $\chi_{\mathrm{AC}}$ and the fit are shown in Fig. $5 \mathrm{~b}$. For the $3 \mu \mathrm{m}$ disk we find a $T_{C}$ of $204.6 \pm 0.2 \mathrm{~K}$, 


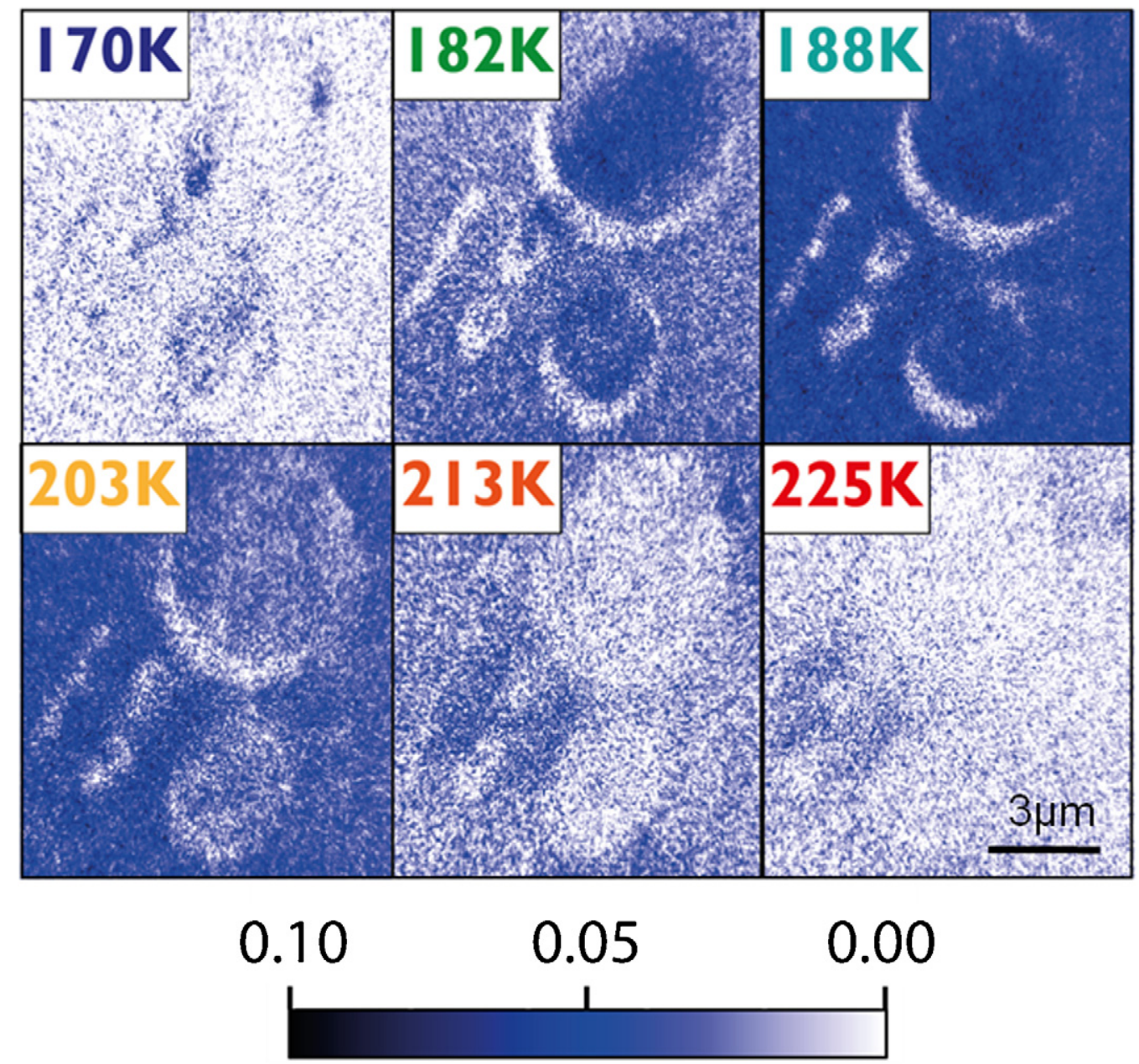

Fig. 4. XPEEM images of the local magnetic susceptibility $\chi_{A C}$ at selected temperatures covering the range of the magnetic phase transition. The local disk pattern is shown in panel (b) of Fig. 3.
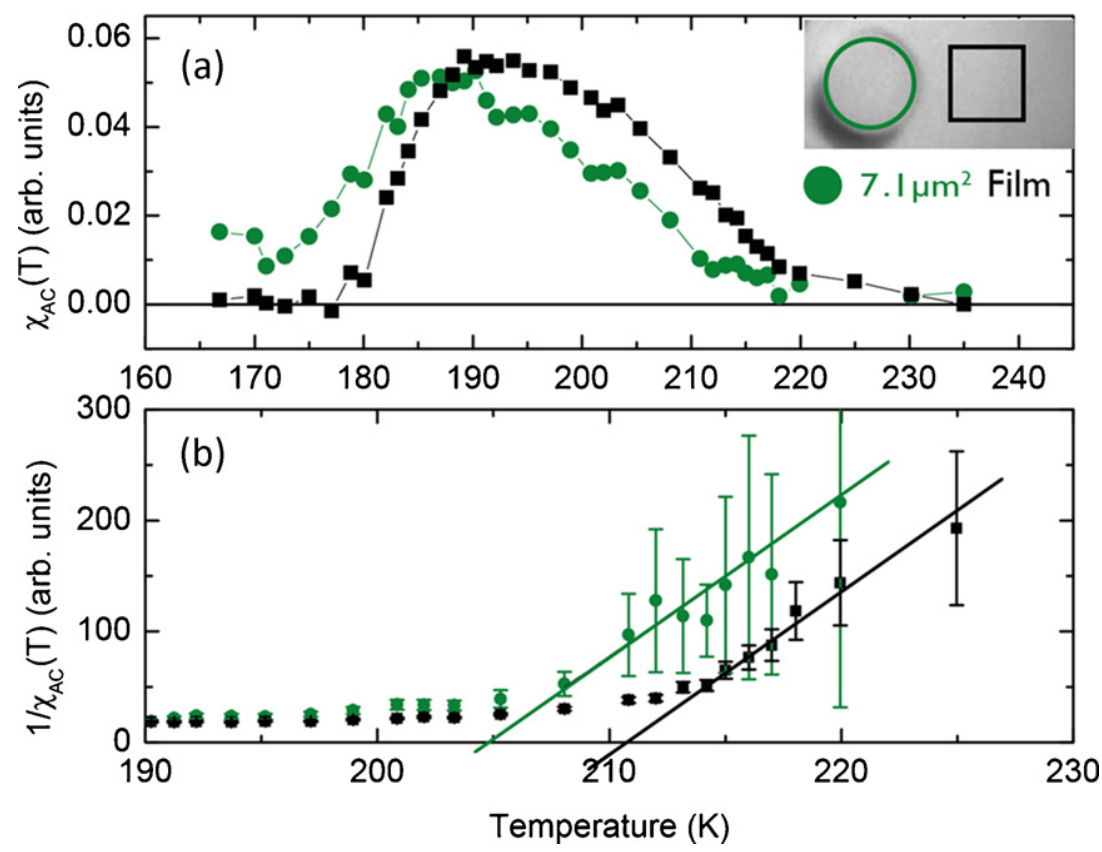

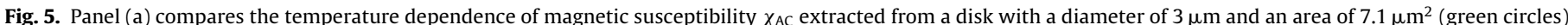

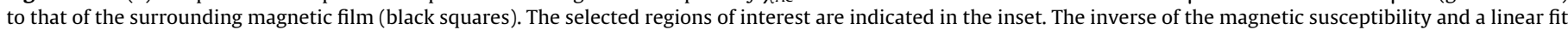

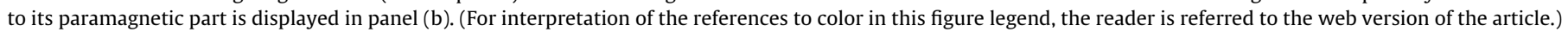


which is $6 \mathrm{~K}$ below the $T_{\mathrm{C}}$ of the continuous film of $210.6 \pm 0.6 \mathrm{~K}$. Even in such rather large structures we find $T_{C}$ significantly lowered compared to a continuous film. This surprising onset of finite size effects on the $\mu \mathrm{m}$ scale might be explained by the divergence of the correlation length at $T_{\mathrm{C}}$. In previous measurements we found a correlation length of about $0.7 \pm 0.1 \mu \mathrm{m}$ at $T_{\mathrm{C}}$ in an Iron film [20]. Long range magnetic fluctuations might cause the reduced $T_{C}$ on the $3 \mu \mathrm{m}$ disk.

\section{Conclusion}

We presented a special sample holder with magnetic field and temperature control. Due to its yoke geometry, confining the magnetic field to a small area, it can be used to apply magnetic fields up to $50 \mathrm{mT}$ during imaging without affecting the spatial resolution of the SPEEM set-up significantly. Imaging under applied magnetic field in combination with temperature control opens new perspectives for the investigation of magnetic micro and nanostructures by XPEEM. We demonstrated the magnetic hysteresis of an individual Fe nanocube at $115 \mathrm{~K}$. Imaging the local magnetic susceptibility $\chi_{\mathrm{AC}}$ of a patterned Iron film we find a surprising onset of finite size effects on the $\mu \mathrm{m}$ scale. Even in structures with lateral extensions in the $\mu \mathrm{m}$ scale we observe a significant reduction of $T_{\mathrm{C}}$ compared to an extended film. In combination with other possibilities such as depth resolved imaging this technique can provide new insights to magnetic nanostructures.

\section{Acknowledgements}

Support by F. Nolting is acknowledged for the design of the sample holder. J. H-A acknowledges financial support by MAT $2011 / 23791$.

\section{References}

[1] E. Bauer, J. Electron Spectrosc. Relat. Phenom. 975 (2001) 114.

[2] C.M. Schneider, G. Schönhense, Rep. Prog. Phys. 65 (2002) 1785.
[3] F. Nolting, A. Scholl, J. Stöhr, J. Fompeyrine, H. Siegwart, J.-P. Locquet, S. Anders, J. Lüning, E.E. Fullerton, M.F. Toney, M.R. Scheinfein, H.A. Padmore, Nature 405 (2000) 767.

[4] H. Ohldag, A. Scholl, F. Nolting, S. Anders, F.U. Hillebrecht, J. Stöhr, Phys. Rev. Lett. 86 (2001) 2878

[5] C.T. Chen, Y.U. Idzerda, H.-J. Lin, N.V. Smith, G. Meigs, E. Chaban, G.H. Ho, E. Pellegrin, F. Sette, Phys. Rev. Lett. 75 (1995) 152.

[6] A. Fraile Rodríguez, A. Kleibert, J. Bansmann, A. Voitkans, L.J. Heyderman, F. Nolting, Phys. Rev. Lett. 104 (2010) 127201.

[7] F. Kronast, N. Friedenberger, K. Ollefs, S. Gliga, L. Tati-Bismaths, R. Thies, A. Ney, R. Weber, C. Hassel, F.M. Römer, A.V. Trunova, C. Wirtz, R. Hertel, H.A. Dürr, M. Farle, Nano Lett. 11 (2011) 1710.

[8] R. Fink, M.R. Weiss, E. Umbach, D. Preikszas, H. Rose, R. Spehr, P. Hartel, W. Engel, R. Degenhardt, R. Wichtendahl, H. Kuhlenbeck, W. Erlebach, K. Ihmann, R. Schlögl, H.-J. Freund, A.M. Bradshaw, G. Lilienkamp, Th. Schmidt, E. Bauer, G. Benner, J. Electron Spectrosc. Relat. Phenom. 84 (1997) 231.

[9] J. Feng, E. Forest, A.A. MacDowell, M. Marcus, H. Padmore, S. Raoux, D. Robin, A. Scholl, R. Schlueter, P. Schmid, J. Stöhr, W. Wan, D.H. Wei, Y. Wu, J. Phys.: Condens. Matter 17 (2005) S1339.

[10] F. Kronast, R. Ovsyannikov, A. Kaiser, C. Wiemann, S.-H. Yang, D.E. Bürgler, R. Schreiber, F. Salmassi, P. Fischer, H.A. Dürr, C.M. Schneider, W. Eberhardt, C.S. Fadley, Appl. Phys. Lett. 93 (2008) 243116.

[11] A.X. Gray, F. Kronast, C. Papp, S.-H. Yang, S. Cramm, I. Krug, F. Salmassi, E. Gullikson, D. Hilken, E. Anderson, P. Fischer, C.M. Schneider, C.S. Fadley, Appl. Phys. Lett. 97 (2010) 062503.

[12] J. Kimling, F. Kronast, S. Martens, T. Böhnert, M. Martens, J. Herrero-Albillos, L. Tati-Bismaths, U. Merkt, K. Nielsch, G. Meier, Phys. Rev. B 84 (2011) 174406.

[13] G. Schneider, P. Guttmann, S. Heim, S. Rehbein, D. Eichert, B. Niemann, AIP Conf. Proc. 879 (2007) 1291-2129.

[14] P. Fischer, T. Eimüller, G. Schütz, G. Bayreuther, S. Tsunashima, N. Takagi, G. Denbeaux, D. Attwood, J. Synchrotron Radiat. 8 (2001) 325-327.

[15] L.J. Heyderman, F. Nolting, D. Backes, S. Czekaj, L. Lopez-Diaz, M. Kläui, U. Rüdiger, C.A.F. Vaz, J.A.C. Bland, R.J. Matelon, U.G. Volkmann, P. Fischer, Phys. Rev. B 73 (2006) 214429.

[16] L. Heyne, M. Kläui, J. Rhensius, L. Le Guyader, F. Nolting, Rev. Sci. Instrum. 81 (2010) 113707.

[17] L. Heyne, J. Rhensius, D. Ilgaz, U. Rüdiger, M. Kläui, L. Joly, F. Nolting, L.J. Heyderman, J.U. Thiele, F. Kronast, Phys. Rev. Lett. 105 (2010) 187203.

[18] A. Gloskovskii, J. Barth, B. Balke, G.H. Fecher, C. Felser, F. Kronast, R. Ovsyannikov, H.A. Dürr, W. Eberhard, G. Schönhense, J. Phys. D: Appl. Phys. 40 (2007) 1570-1575.

[19] F. Kronast, J. Schlichting, F. Radu, S.K. Mishra, T. Noll, H.A. Dürr, Surf. Interface Anal. 42 (2010) 1532.

[20] M. Römer, F. Kronast, L. Heyne, C. Hassel, A. Banholzer, M. Kläui, R. Meckenstock J. Lindner, M. Farle, Appl. Phys. Lett. 96 (2010) 122501.

[21] M.R. Scheinfein, K.E. Schmidt, K.R. Heim, G.G. Hembree, Phys. Rev. Lett. 76 (1996) 1541-1544.

[22] Y. Li, M. Farle, K. Baberschke, Phys. Rev. B: Rapid Commun. 41 (1990) 9596. 